\title{
Thermotropic liquid crystalline polyesters derived from 2-chloro hydroquinone
}

\author{
NAGESH MANURKAR, SAYAJI MORE, KHUDBUDIN MULANI, NITIN GANJAVE \\ and NAYAKU CHAVAN* \\ Polymer Science and Engineering Division, CSIR-National Chemical Laboratory, Dr. Homi Bhabha Road, \\ Pashan, Pune, Maharashtra 411 008, India \\ E-mail: nn.chavan@ncl.res.in
}

MS received 6 March 2017; revised 22 June 2017; accepted 5 July 2017; published online 24 August 2017

\begin{abstract}
Synthesis of thermotropic liquid crystalline polyesters derived from bis[4-hydroxy benzoyloxy]2-chloro-1,4-benzene (BHBOCB) and aliphatic dicarboxylic acid chlorides by interfacial polycondensation methodology is presented. Synthesised polyesters consist of bis[4-hydroxy benzoyloxy]-2-chloro-1,4-benzene as a mesogen and aliphatic diacid chloride as flexible spacer. The length of oligomethylene units in the polymer was varied from the trimethylene to the dodecamethylene groups. Synthesized polyesters were characterized by differential scanning calorimetry and optical microscopy. The transition temperatures and thermodynamic properties were studied for all these polymers. These polyesters exhibited thermotropic liquid crystalline behavior and showed nematic texture except decamethylene spacer. Decamethylene spacer based polyester showed marble texture of smectic C. Mesophase stability of these polyesters was higher than $123^{\circ} \mathrm{C}$ (except first heating cycle of PE-1).
\end{abstract}

Keywords. Thermotropic liquid crystalline polyesters; aromatic mesogen; aliphatic flexible spacers.

\section{Introduction}

The synthesis of thermotropic liquid crystalline aromatic polyesters (TLCP) have received more attention in the field of engineering polymers due to their attractive properties such as low melt viscosities, thermal endurance, chemical stability and excellent mechanical properties. Liquid crystalline polymers (LCP's) are extremely useful for various applications including fabrication of numerous optical and electronic devices, flame retardance and solvent resistance. ${ }^{1-5}$ The thermotropic liquid crystalline behavior of polymers is of strong current interest among polymer scientists, because of their outstanding thermal and chemical resistance, high strength as well as low linear viscosity in liquid crystalline state. ${ }^{6-13}$ Basically, thermotropic liquid crystalline polymers are of two types. In the first type, aliphatic flexible spacer incorporated into the main chain to reduce transition temperature as well as rigidity and improve the solubility, whereas in the second

\footnotetext{
*For correspondence
}

type, flexible spacers are incorporated in side chain as substituent.

In TLCP, the substituent effects are of great importance because crystal to liquid crystal transition temperatures of fully extended rigid rod polymers are extremely high. Thus, it is desirable to lower the crystal to liquid crystal transition temperature of liquid crystalline polymers from processing point of view. Kleinschuster et al., first reported the effect of alkyl substitution and the use of monosubstituted hydroquinones in random copolyesters results in a considerable decrease in the crystal to liquid crystal transition temperature and permits melt spinning from the liquid crystalline states. ${ }^{14}$ Lenz et al., described the effect of alkyl substitution on mesogenic unit with respect to glass transition temperature, melting temperature, thermal stabilities, etc. and inferred that polymer with alkyl substitution more than eight carbon atoms or longer does not form a liquid crystalline phase. ${ }^{15}$

Over the last few decades, several strategies have been used to lower the liquid crystalline phase transition temperature and one of the most popular strategies 
used for this purpose is incorporation of substituent on mesogenic unit. Since melting temperatures of fully extended rigid rod polymers are very high, majority of such polymers degrade before its isotropization temperature. Substitution lowers phase transition temperature substantially due to decrease in axial ratio of rigid mesogenic unit. So, it is desirable to lower the melting temperature of processing by conventional methods. This could be accomplished by introducing substituent on the mesogenic unit of the polymer. ${ }^{16}$ In our previous papers, we reported the synthesis and characterization of thermotropic liquid crystalline polyesters derived from hydroquinone and methyl hydroquinone-based rigid rod diols as mesogenic units and aliphatic dicarboxylic acids as flexible spacers. ${ }^{17,18}$

The present investigation demonstrates synthesis and characterisation of TLCP derived from bis[4-hydroxy benzoyloxy]-2-chloro-1,4-benzene (BHBOCB) as mesogenic moiety and aliphatic diacid chloride as flexible spacer. Flexible spacer was inserted into the backbone of the rigid polymer to dissociate the greater order of the main-chain and decouple the motion of mesogenic moiety from polymer backbone. We also describe synthesis, thermal transition temperatures, thermodynamic properties such as entropy/enthalpy, mesomorphic range and morphology in the mesophase of the liquid crystalline polymers.

\section{Experimental}

\subsection{Materials}

2-Chloro hydroquinone, 4-hydroxy benzoic acid, dibasic acids such as glutaric, adipic, pimelic, suberic, azelaic, sebacic and dodecanedioic were purchased from Aldrich. 2Chloro hydroquinone and 4-hydroxy benzoic acid were purified by recrystallisation from acetone and hot distilled water, respectively. Acetic anhydride, dichloromethane, methanol, pyridine, chloroform, thionyl chloride, pet ether and potassium hydroxide were procured from Merck and were used as received.

\subsection{Synthesis of trimesogenic diol [BHBOCB]}

Bis[4-hydroxy benzoyloxy]-2-chloro-1,4-benzene [trimesogen] was synthesized from p-hydroxy benzoic acid as starting material and 2-chloro-hydroquinone as central moiety as reported in our earlier studies. ${ }^{19}$ The synthesis route of BHBOCB trimesogen is depicted in Scheme 1 and thermotropic liquid crystalline polyesters were synthesized according to Scheme 2.

2.2a 4-Acetoxy benzoic acid [4-ACBA]: In a $500 \mathrm{~mL}$ beaker, 4-hydroxy benzoic acid (13.81g, 0.1 mole) and distilled water $(250 \mathrm{~mL})$ were added and stirred to make uniform slurry. In another flask, sodium hydroxide ( $4.4 \mathrm{~g}, 0.11$ mole) was dissolved in $50 \mathrm{~mL}$ distilled water and was added to

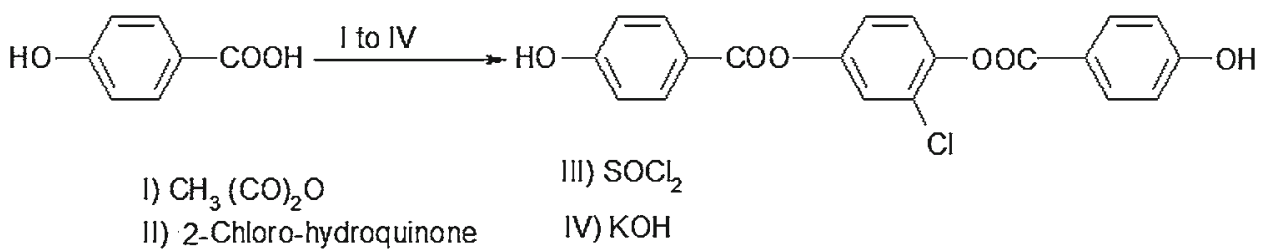

Scheme 1. Synthesis of bis-(4-hydroxy benzoyloxy)-2-chloro-1,4-benzene (BHBOCB).
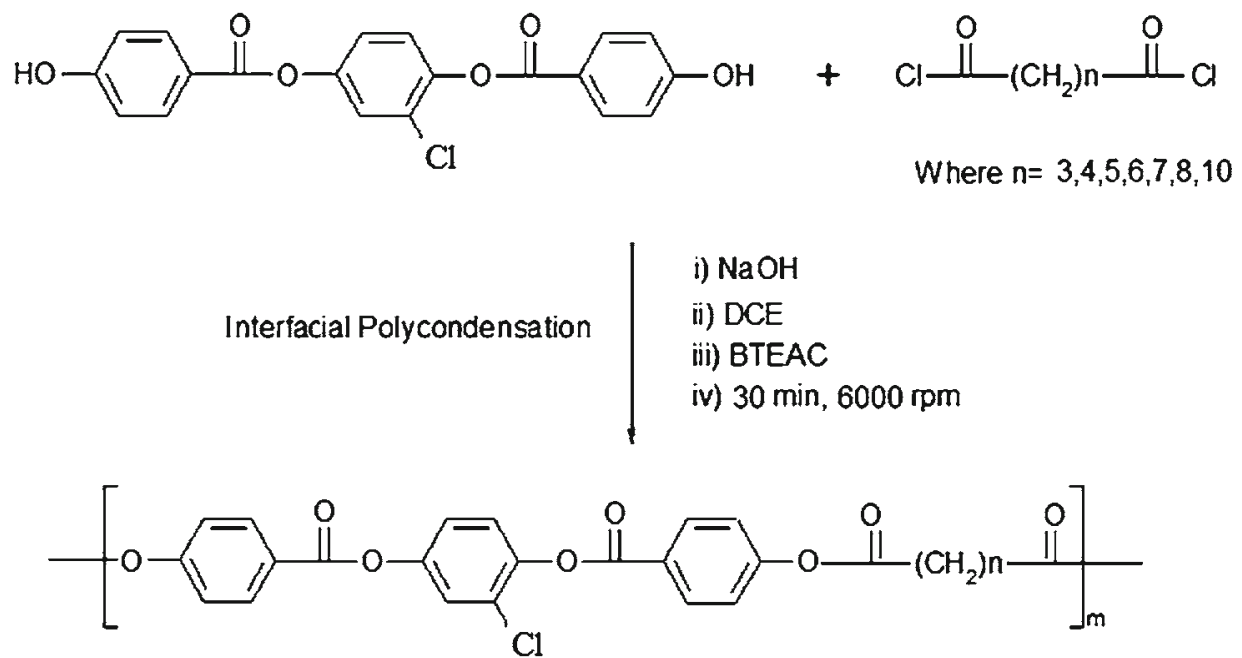

Scheme 2. Synthesis of polyesters. 
the slurry. The reaction mixture was stirred till 4-hydroxy benzoic acid dissolved completely, and to it distilled acetic anhydride (11.23 g, 0.11 mole) was added slowly. After the addition of acetic anhydride, stirring was continued for $4 \mathrm{~h}$ at room temperature to obtain a precipitate of 4-acetoxy benzoic acid. The precipitate obtained was filtered and washed several times with cold dilute hydrochloric acid and distilled water. The crude product was recrystallized from methanol and dried under reduced pressure. Yield: $80 \%$. M.p.: $190^{\circ} \mathrm{C}$, IR (Nujol, $\left.\mathbf{c m}^{-1}\right): 1730$ (C=O stretching); ${ }^{1} \mathrm{H}$ NMR (DMSO$\left.\mathrm{d}_{6, \delta}\right): 8.00(\mathrm{~d}, 2 \mathrm{H}) ; 7.30(2 \mathrm{H}, \mathrm{d}) ; 2.30(\mathrm{~s}, 3 \mathrm{H}) ; 10.10(\mathrm{~s}, 1 \mathrm{H})$.

2.2b 4-Acetoxy benzoyl chloride: 4-Acetoxy benzoic acid $(9.0 \mathrm{~g}, 0.05$ mole) was placed in a single necked $100 \mathrm{~mL}$ round bottom flask. To this flask, thionyl chloride $(5 \mathrm{~mL}, 0.07$ mole) was added drop wise and refluxed the reaction mixture gently for $8 \mathrm{~h}$. The initial heterogeneous mass was homogenized. Excess thionyl chloride was removed by distillation. The crude acid chloride was purified by double distillation under reduced pressure. Yield: $90 \%$. M.p.: $29^{\circ}$ C. IR (Nujol, $\left.\mathrm{cm}^{-1}\right)$ : $1780(\mathrm{COCl}), 1730(\mathrm{C}=\mathrm{O}$ stretching $)$.

\section{2c Bis [4-acetoxy benzoyloxy]-2-chloro-1,4-} benzene: Three-necked $500 \mathrm{~mL}$ round bottom flask was equipped with magnetic stirrer, nitrogen inlet and calcium chloride guard tube. To the reaction flask, 2-chloro hydroquinone $(5.78 \mathrm{~g}, 0.04$ mole) and pyridine $(25 \mathrm{~mL}, 0.3$ mole $)$ were added and stirred till dissolution. In another flask, 4acetoxy benzoyl chloride (23.82 g, 0.12 mole) and $300 \mathrm{~mL}$ dry 1,2-dichloroethane were placed and the acid chloride solution was added to the reaction mixture of 2-chlorohydroquinone solution. This reaction mixture was stirred under nitrogen blanket for $48 \mathrm{~h}$ at room temperature. The reaction mixture was then washed sequentially with $5 \%$ sodium carbonate solution, $5 \%$ hydrochloric acid and distilled water. 1,2-Dichloroethane layer was evaporated to dryness on rotavapor. The crude product obtained was recrystallised from chloroform/petroleum ether [60:80] solvent mixture and dried in vacuum oven at $80^{\circ} \mathrm{C}$ for 4 h. Yield $90 \%$. M. p.: $181^{\circ} \mathrm{C}$, IR (Nujol, $\left.\mathrm{cm}^{-1}\right)$ : 1740 (C=O stretching), 760 (C-H bending). ${ }^{1} \mathrm{H} \mathrm{NMR}\left(\mathrm{CDCl}_{3}, \delta\right): 2.3(\mathrm{~s}, 6 \mathrm{H}) ; 7.1(\mathrm{~m}, 6 \mathrm{H}) ; 7.3(\mathrm{~d}, 1 \mathrm{H}) ; 8.2$ (dd, $4 \mathrm{H})$.

\section{2d Bis[4-hydroxy benzoyl oxy]-2-chloro-1,4-} benzene [BHBOCB]: Single-necked $250 \mathrm{~mL}$ round bottom flask was equipped with magnetic stirrer and ice bath. To the reaction flask, potassium hydroxide (1.32 g, 0.02 mole) and $100 \mathrm{~mL}$ methanol were placed, stirred till dissolution and the solution was cooled to $0-5^{\circ} \mathrm{C}$. To the alcoholic potassium hydroxide solution, bis[4-acetoxy benzoyloxy]2-chloro-1,4-benzene (4.34 g, 0.01mole) was added. The reaction mixture was stirred at $0-5^{\circ} \mathrm{C}$ for $4 \mathrm{~h}$ and then acidified with $4 \mathrm{~N}$ hydrochloric acid. Methanol was removed under reduced pressure. The crude product obtained was dissolved in $400 \mathrm{~mL}$ ethyl acetate and ethyl acetate layer was washed three times with distilled water. Ethyl acetate was distilled off and the solid was extracted with chloroform. Chloroform was distilled off to get solid trimesogenic diol product. This solid product was washed with methanol and dried under reduced pressure at $80^{\circ} \mathrm{C}$ for $4 \mathrm{~h}$. Yield: 75\%. M.p.: $296^{\circ}$ C. IR (Nujol, $\mathrm{cm}^{-1}$ ): 3393 (OH stretching), 3072 (C-H stretching), 1907 (anti symmetric $\mathrm{C}=\mathrm{C}$ stretching), 1708 ( $\mathrm{C}=\mathrm{O}$ stretching), 1609 and 1591 (aromatic $\mathrm{C}=\mathrm{C}$ stretching), 1512 (C-H bending), 1447 (symmetric $-\mathrm{C}=\mathrm{C}$ stretching), 1276, 1162 (symmetric $-\mathrm{C}=\mathrm{O}$ stretching), 1066, 762 (C-Cl substitution) and $846 \mathrm{~cm}^{-1}$ (parasubstitution). ${ }^{1} \mathrm{H}$ NMR (DMSO- $\left.d_{6}\right): \delta 6.94-6.98(\mathrm{a}, \mathrm{dd}, 4 \mathrm{H})$, 7.34-7.51 (b, dd, 3H), 7.96 (c, S, 1H), 8.00-8.04 (d, dd, 4H), 10.64(e, s, $2 \mathrm{H}) .{ }^{13} \mathrm{C}$ NMR (DMSO- $d_{6}$ ) $: \delta 115.86,122.44-$ $126.58,132.71,144.70,148.87,163.29,164.29$.

\section{2e Preparation of aliphatic diacid chlorides: Glu-} taroyl chloride, adipoyl chloride, pimeloyl chloride, suberoyl chloride, azeloyl chloride, sebacoyl chloride and dodecanedioyl chloride were synthesized from the parent diacids. The aliphatic diacids were gently refluxed with thionyl chloride at $80^{\circ} \mathrm{C}$ for $8 \mathrm{~h}$ till a clear solution was obtained. The acid chloride solution was initially distilled under lower reduced pressure (100-150 $\mathrm{mm} \mathrm{Hg}$ ) to remove the excess of thionyl chloride and was double distilled under reduced pressure (1-2 $\mathrm{mm} \mathrm{Hg}$ ) at $150^{\circ} \mathrm{C}$ to get pure acid chloride, prior to use.

\subsection{Polycondensation}

2.3a Interfacial polycondensation: Recipe used for interfacial polycondensation is presented in Table 1. Twonecked $100 \mathrm{~mL}$ round bottom flask was equipped with mechanical stirrer, nitrogen inlet, and calcium chloride guard tube. To the reaction flask, trimesogen BHBOCB (1.539 $\mathrm{g}, 0.04$ mole), sodium hydroxide [0.008 mole], benzyl triethyl ammonium chloride $(0.02 \mathrm{~g})$ and $15 \mathrm{~mL}$ distilled water were added and stirred for $5 \mathrm{~min}$. Into the another $100 \mathrm{~mL}$ stoppered flask, 0.04 mole of acid chloride and $15 \mathrm{~mL}$ dry 1,2-dichloroethane were placed and stirred for $5 \mathrm{~min}$. The acid chloride solution was added into the reaction flask. The reaction mixture was vigorously stirred at 6000-7000 rpm for $30 \mathrm{~min}$ at $0-5^{\circ} \mathrm{C}$. The polyester was precipitated by pouring it into $500 \mathrm{~mL}$ methanol. The solution was filtered and

Table 1. Recipe of BHBOCB and aliphatic diacid chlorides for interfacial polycondensation.

Polymer Code Aliphatic diacid chloride Polyester [m/ru]*

\begin{tabular}{lcc}
\hline PE-1 & Glutaryl & 480.83 \\
PE-2 & Adipoyl & 494.86 \\
PE-3 & Pimeloyl & 508.88 \\
PE-4 & Suberoyl & 522.91 \\
PE-5 & Azeloyl & 536.94 \\
PE-6 & Sebacoyl & 550.96 \\
PE-7 & Dodecyl & 579.01 \\
\hline
\end{tabular}

* mole per repeat unit. 
Table 2. Transition temperature and thermodynamic data of BHBOCB polyesters (First heating cycle).

\begin{tabular}{lccccccccc}
\hline Polymer Code & {$\left[\mathrm{CH}_{2}\right]_{n} \mathbf{n}$} & $\mathbf{K}-\mathbf{N}\left({ }^{\circ} \mathbf{C}\right)$ & $\mathbf{N}-\mathbf{I}\left({ }^{\circ} \mathbf{C}\right)$ & $\Delta \mathbf{T}\left({ }^{\circ} \mathbf{C}\right)$ & $\Delta \mathbf{H} \mathbf{K}-\mathbf{N}$ & $\Delta \mathbf{H} \mathbf{N}-\mathbf{I}$ & $\Delta \mathbf{S}$ K-N & $\Delta \mathbf{S}$ N-I & DOC \% \\
\hline PE-1 & 3 & 217.5 & 278.1 & 60.6 & 4.52 & 6.35 & 9.22 & 11.52 & 40.81 \\
PE-2 & 4 & 224.9 & 345.9 & 121.0 & 6.88 & 4.45 & 13.81 & 7.19 & 40.00 \\
PE-3 & 5 & 170.0 & 278.2 & 108.2 & 6.62 & 5.24 & 14.95 & 9.50 & 46.07 \\
PE-4 & 6 & 173.6 & 303.8 & 130.2 & 1.68 & 3.09 & 3.76 & 5.36 & 37.50 \\
PE-5 & 7 & 173.2 & 261.5 & 88.3 & 12.30 & 3.40 & 27.57 & 6.36 & 50.00 \\
PE-6 & 8 & 155.2 & 283.8 & 128.6 & 2.65 & 3.47 & 6.19 & 6.24 & 40.42 \\
PE-7 & 10 & $120.4^{\mathrm{a}}$ & $204.8^{\mathrm{b}}$ & 84.4 & $3.42^{\mathrm{a}}$ & $2.90^{\mathrm{b}}$ & $8.70^{\mathrm{a}}$ & $6.07^{\mathrm{b}}$ & 48.13 \\
\hline
\end{tabular}

K-N $\left({ }^{\circ} \mathrm{C}\right)$ : Crystal - liquid crystal transition temperature, N-I $\left({ }^{\circ} \mathrm{C}\right)$ : Liquid crystal - isotropic transition temperature, $\Delta \mathrm{T}\left({ }^{\circ} \mathrm{C}\right)$ : Temperature range of mesophase stability, $\Delta \mathrm{H}(\mathrm{K}-\mathrm{N})$ and $\Delta \mathrm{H}(\mathrm{N}-\mathrm{I})$ : Enthalpy change in $\mathrm{kJ} \mathrm{mol}^{-1}$ of repeat unit (mru), $\Delta \mathrm{S}(\mathrm{K}-\mathrm{N})$ and $\Delta \mathrm{S}(\mathrm{N}-\mathrm{I})$ : Entropy change in Joule $\mathrm{mru}^{-1}(\mathrm{~K})$, DOC: Percent degree of crystallinity. ${ }^{\mathrm{a}}$ Crystal to smectic transition; ${ }^{\mathrm{b}} \mathrm{Smectic}$ to isotropic transition.

Table 3. Transition temperature and thermodynamic data of BHBOCB polyesters (Second heating cycle).

\begin{tabular}{lccccccccc}
\hline Polymer Code & {$\left[\mathrm{CH}_{\mathbf{2}}\right]_{\mathbf{n}} \mathbf{n}$} & $\mathbf{K}-\mathbf{N}\left({ }^{\circ} \mathbf{C}\right)$ & $\mathbf{N}-\mathbf{I}\left({ }^{\circ} \mathbf{C}\right)$ & $\Delta \mathbf{T}\left({ }^{\circ} \mathbf{C}\right)$ & $\Delta \mathbf{H ~ K - N}$ & $\Delta \mathbf{H}$ N-I & $\Delta \mathbf{S} \mathbf{K}-\mathbf{N}$ & $\Delta \mathbf{S}$ N-I & DOC $\%$ \\
\hline PE-1 & 3 & 162.9 & 341.3 & 178.4 & 2.12 & 5.72 & 4.86 & 9.32 & 40.81 \\
PE-2 & 4 & 178.8 & 367.7 & 188.9 & 5.88 & 9.80 & 13.04 & 15.30 & 40.00 \\
PE-3 & 5 & 159.6 & 348.2 & 188.5 & 3.10 & 6.92 & 7.18 & 11.15 & 46.07 \\
PE-4 & 6 & 139.6 & 337.8 & 198.4 & 0.73 & 8.74 & 1.78 & 25.85 & 37.50 \\
PE-5 & 7 & 166.0 & 282.8 & 116.8 & 5.37 & 4.67 & 12.23 & 8.41 & 50.00 \\
PE-6 & 8 & 162.8 & 303.8 & 141.0 & 5.06 & 6.34 & 11.63 & 10.63 & 40.42 \\
PE-7 & 10 & $104.3^{\mathrm{a}}$ & $227.3^{\mathrm{b}}$ & 123.0 & $0.70^{\mathrm{a}}$ & $2.44^{\mathrm{b}}$ & $1.85^{\mathrm{a}}$ & $4.87^{\mathrm{b}}$ & 48.13
\end{tabular}

$\mathrm{K}-\mathrm{N}\left({ }^{\circ} \mathrm{C}\right)$ : Crystal - liquid crystal transition temperature, N-I $\left({ }^{\circ} \mathrm{C}\right)$ : Liquid crystal - isotropic transition temperature, $\Delta \mathrm{T}\left({ }^{\circ} \mathrm{C}\right)$ : Temperature range of mesophase stability, $\Delta \mathrm{H}(\mathrm{K}-\mathrm{N})$ and $\Delta \mathrm{H}(\mathrm{N}-\mathrm{I})$ : Enthalpy change in $\mathrm{kJ} \mathrm{mol}^{-1}$ of repeat unit (mru), $\Delta \mathrm{S}(\mathrm{K}-\mathrm{N})$ and $\Delta \mathrm{S}$ (N-I): Entropy change in Joule $\mathrm{mru}^{-1}$ (K), DOC: Percent degree of crystallinity. ${ }^{\mathrm{a}}$ Crystal to smectic transition; ${ }^{\mathrm{b}}$ Smectic to isotropic transition.

the obtained solid polymer was washed with 5 weight percent sodium carbonate solution, dilute hydrochloric acid and distilled water. The obtained polymer was further purified by soxhlet extraction using methanol as solvent and dried under reduced pressure at $80^{\circ} \mathrm{C}$ for $8 \mathrm{~h}$.

\subsection{Characterization}

Synthesized polymers were soluble in chlorinated organic solvents such as chloroform, carbon tetrachloride, dichloromethane, dichloroethane, 4-chlorophenol or phenol:TCE $(50: 50 \mathrm{v} / \mathrm{v})$ mixture. The thermal properties were determined with a Mettler DSC-30 thermal analyzer under a nitrogen atmosphere. Indium and solid mixture of Indium-Lead-Zinc were used as calibration standards for enthalpy $(\Delta \mathrm{H})$ calculation and temperature scale, respectively. The endotherm peaks (maxima) were taken as phase transition temperatures. The enthalpies and entropies of liquid crystalline phase transitions were determined. A Leitz Ortholux polarizing microscope with a Mettler FP-52 hot stage controlled by Mettler FP-5 temperature controller was used for visual examination of phase changes in the polymers. Wide angle X-ray diffractometer was used to determine the degree of crystallinity at room temperature. Inherent viscosities were determined with $0.5 \%$ solution in chloroform at $30^{\circ} \mathrm{C}$.

\section{Results and Discussion}

\subsection{General properties}

The transition temperatures and thermodynamic properties of polyesters are presented in Tables 2 and 3.

All the polyesters exhibit major two endothermic melting peaks which correspond to the crystal to liquid crystal and liquid crystal to the isotropic transitions along with small melting peaks during the first heating cycle. This is due to solid-solid transitions, formed in rapid succession when more than one liquid crystalline phase is present. All the polyesters exhibited a rather narrow endothermic transition for the mesophase at the isotropization temperature, which suggests that polydispersity was rather narrow. To eliminate the thermal history of the polyester samples generated during solvent evaporation and drying, the second DSC heating was applied for all polyester samples to determine the 
melting transitions of the liquid crystalline polymers. In the second heating cycle, all polyesters display two small endothermic peaks which indicate that thermal history of polyester sample is eliminated.

Synthesized polyesters are well ordered and soluble in the polymerization solvent medium. A broadening of isotropic transition may be due to polydispersity, as indeed observed for the polyesters based on unsubstituted trimesogen, wherein insolubility intervened. The peak maxima were selected to make comparative estimation of structure-property relationship.

No definite trend was observed in crystal to liquid crystal transition temperature with respect to the number of methyelene units (n) incorporated in aliphatic spacer, but crystal to liquid crystal or liquid crystal to isotropic transition temperature decreases with number of methylene units due to the dilution of mesogen or decrease in axial ratio (Table 2). Mesophase stability of hydroquinone, methyl hydroquinone and chlorohydroquinone-based polyesters showed significant difference in mesophase stability on the basis of second heating cycle in which thermal history was eliminated. ${ }^{17,18}$ Mesophase stability of the substituted hydroquinone (e.g., methyl or chloro)-based rigid-rod flexible spacer polymers showed higher than unsusbtituted polymers on the basis of second heating cycle. Maximum mesophase stabilities were observed 198.4, 163.1 and $107.8^{\circ} \mathrm{C}$ for chloro, methyl and unsubstituted hydroquinone-based polymers, respectively, whereas minimum mesophase stabilities were observed 123.0, 89.8 and $99.8^{\circ} \mathrm{C}$ for chloro, methyl and unsubstituted hydroquinone-based polymers, respectively. It shows a considerable decrease in crystal to liquid crystal transition temperature but no decrease in liquid crystal to isotropic temperature. This may be due to the difference in the pKa values of hydroquinone and chlorohydroquinone which are 10.2 and 8.6, respectively. It clearly indicates that chlorohydroquinone posseses higher acidity than hydroquinone as such.

It is known that deisotropization in main liquid crystalline polyesters exhibit a smaller degree of super cooling. In other words, isotropization is thermodynamically more of an equilibrium (reversible) process, rather than crystal to liquid crystal transition. Due to this effect, crystal to liquid crystal temperature of polyesters was decreased by $50^{\circ} \mathrm{C}$ and liquid crystal to isotropic temperature of polyesters was increased by 25 to $50^{\circ} \mathrm{C}$. Thus, mesophasic stability of polyesters was considerably higher $\left(>120^{\circ} \mathrm{C}\right)$.

All polyesters, except PE-7, display the formation of nematic schlieren texture from the isotropic phase to liquid crystalline phase upon cooling under polarizing microscope. Polyester PE-7, wherein 10 methyelene

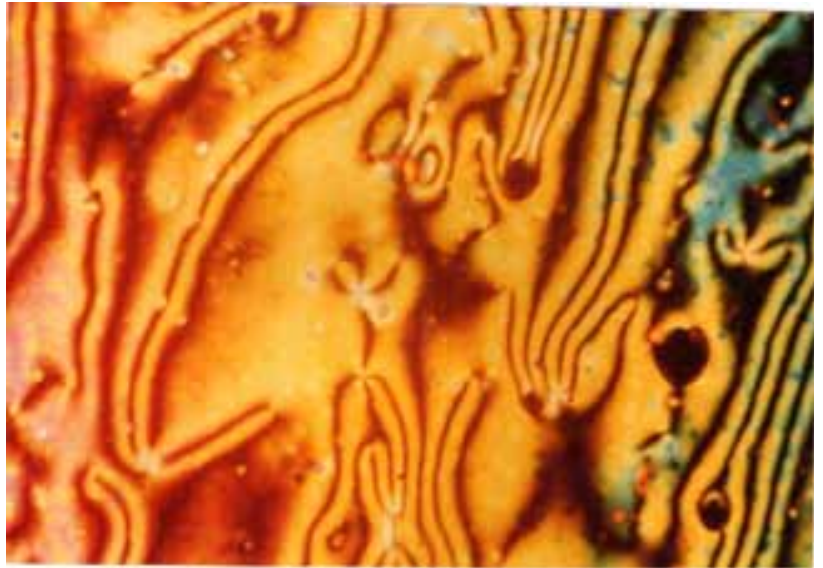

Figure 1. Photomicrograph of polyester PE- 7 at $187.7^{\circ} \mathrm{C}$ observed on cooling from isotropic state.

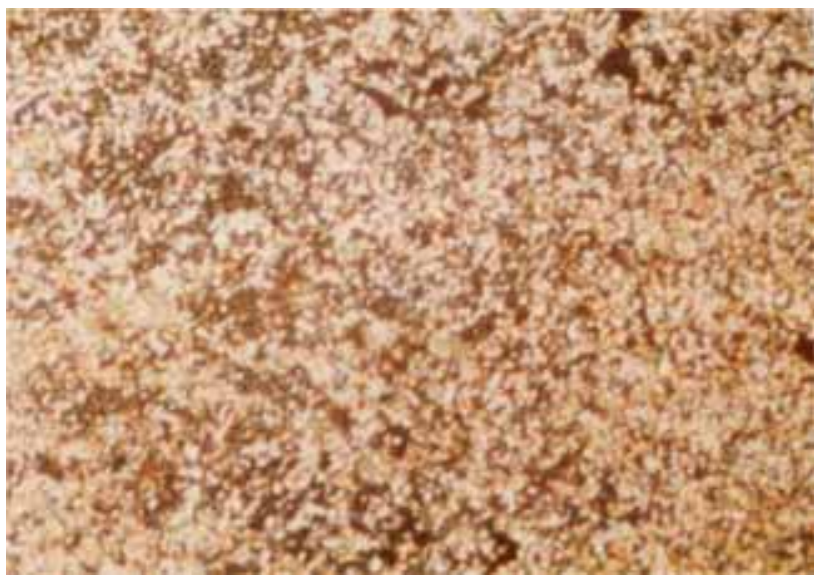

Figure 2. Photomicrograph of polyester PE-7 at $134.8^{\circ} \mathrm{C}$ observed on cooling from isotropic state.

units are linked in the main chain of repeating unit of the polymer, displays the nematic schlieren texture from the isotropic phase to liquid crystalline phase on cooling under polarizing microscope at $187.7^{\circ} \mathrm{C}$ as shown in Figure 1 , and on further cooling showed small droplets of texture of smectic $\mathrm{C}$ at $134.8^{\circ} \mathrm{C}$ under crossed polarizer as seen in Figure 2. ${ }^{20}$ This is because when the number of methylene units is more than 8 side chain crystallization occurs and thus, nematic liquid crystal phase turns to more ordered smectic phase.

\subsection{Substitution effects}

Effect of different substituents on liquid crystalline behaviour of low-molecular mass liquid crystals has been systematically described by Gray ${ }^{21-23}$ and others. ${ }^{16,24,25}$ It is desirable to lower the melting temperature of polymers for processing point of view. This is accomplished by introducing the substituent into mesogenic unit of thermotropic polymers. The first description 
of substituted main chain thermotropic liquid crystalline polymers was made by Roviello and Sirigu. ${ }^{16}$ However, the highly polar substituents $(-\mathrm{Cl},-\mathrm{Br},-\mathrm{CN}$, $-\mathrm{NO}_{2}$ ) are known to be very effective in depressing the crystal to liquid crystal transition temperature. This partial decrease in transition temperature arises from steric effects, which limits the molecular packing efficiency in both crystalline and liquid crystalline states. The Van der Waals radii of chloro, bromo, cyano and nitro substituents are larger than that of methyl group.

Chloro substitution based liquid crystalline polymers significantly lower the transition temperature compared to the unsubstituted polymers. The reduction in crystal to liquid crystal transition temperatures of chlorohydroquinone based thermotropic liquid crystalline polyesters may be due to bulkiness of the chloro substituent compared to the corresponding polyesters prepared from unsubstituted or methyl substituted series. It can be confirmed by bond lengths of C-H, C-Me and C-Cl are 1.07, 1.54 and $1.76 \AA$, respectively. ${ }^{23}$

Substitution plays important role on mesophase stability. If the substituent is electron-withdrawing group such as chloro may substantially change acidity of the resulting polyesters. The ionization constant of chlorohydroquinone is several times greater than that of unsubstituted hydroquinone, which greatly stabilizes the phenoxide ion by permitting a portion of the negative charge to be carried by its own oxygen. Due to this effect, reduction in melting transitions of liquid crystalline phases was observed but isotropization temperatures were not affected. Thus, mesomorphic stability of chlorohydroquinone based liquid crystalline polymers was higher than unsubstituted and methyl substituted hydroquinone based liquid crystalline polymers.

In our previous communication, we have reported that synthesis and thermal properties of polyesters derived from methyl substituted hydroquinone as central moiety. The incorporation of methyl substituent on hydroquinone central moiety of triad mesogen unit resulted in lower isotropisation temperature with respect to the similar number of methylene units. ${ }^{18}$ In short, crystal to liquid crystal transition temperatures of unsubstituted hydroquinone based polyesters were higher than those of the methyl and chlorohydroquinone based polyesters. The trend of crystal to liquid crystal transition temperature of polyesters based on hydroquinone is as follows: $\mathrm{H}>\mathrm{Me}>\mathrm{Cl}$.

\subsection{Thermodynamics of LC States}

The differential scanning calorimetry (DSC) thermograms corresponding to the first and second heating

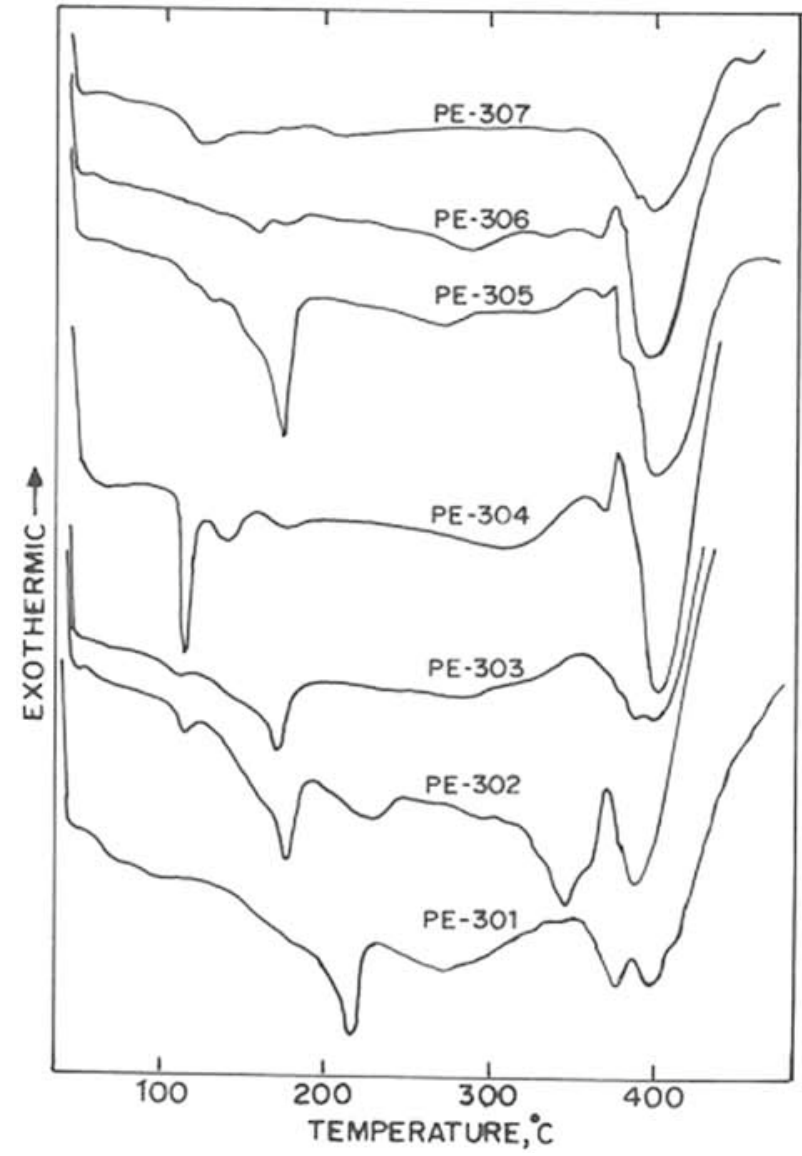

Figure 3. DSC thermograms of BHBOCB polyesters (First heating cycle).

cycles of these polyesters are shown in Figures 3 and 4. The transition temperatures and thermodynamic data, as determined by DSC thermograms, corresponding to the first and second heating cycles of synthesized polyesters are presented in Tables 2 and 3. Looking at the liquid crystal transition temperatures, it can be stated that the odd-even effect is weakly expressed in this homologues series of polyesters.

Rath and Ponrathnam described the nature of lateral substituent on the mesogen has very marginal influence on $\mathrm{T}_{\mathrm{m}}$ in random thermotropic liquid crystalline polyesters. ${ }^{26}$ Ordered polyesters with bulkier substituent on the mesogen usually show greater depression in $\mathrm{T}_{\mathrm{m}} \cdot{ }^{27}$ The attraction due to greater electronegativity of chlorine is outweighed by steric repulsion in ordered systems and resulted in a reduced lateral packing of chains. The greater effect of steric repulsion is not easily realized in randomized structures. Unlike the methyl series, the ordered chloro series shows some odd fluctuations in isotropic transition temperatures $\mathrm{T}_{\mathrm{i}} \cdot{ }^{26}$ In general, these temperatures are lower than those in methylene homologues. Partial decomposition during isotropization and non-equilibrium nature of crystal to 


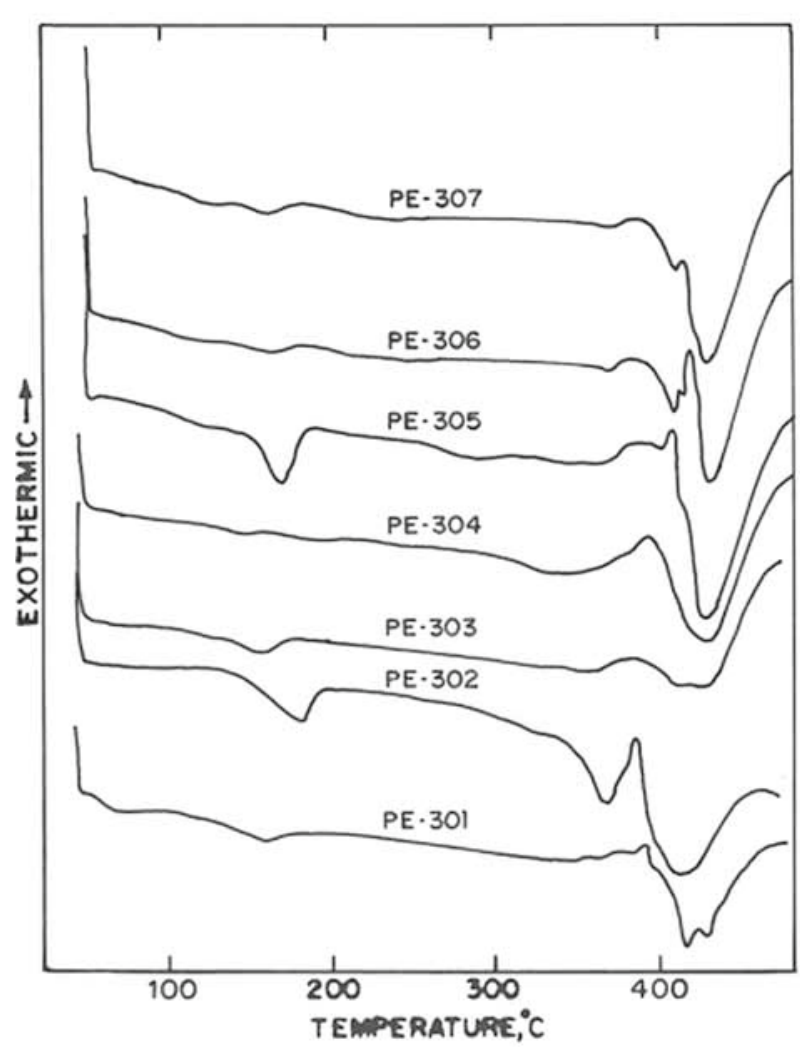

Figure 4. DSC thermograms of BHBOCB polyesters (Second heating cycle).

liquid crystal or liquid crystal to crystal transitions are the probable reasons for the non-emergence of odd-even effect in a clear-cut fashion during isotropic and liquid crystal transitions, respectively.

The aliphatic methylene spacer is expected to give more conformational freedom to the mesogenic units to form ordered structure in melt. Jin et al., synthesised a series of polyesters using biphenyl p-oxybenzoate as a mesogenic unit and pentamethylene as spacer. They observed that presence of the spacer enchances the heat capacity of the compound to form a mesophase. ${ }^{27}$ In general, lowering of the transition temperature occurs due to incorporation of aliphatic spacers. Therefore, the polyesters with the increasing length of the spacer, a downward trend in the clearing temperature was observed. ${ }^{28}$

\subsection{Enthalpy and entropy changes}

The enthalpy and entropy changes for the crystal to liquid-crystal and liquid-crystal to isotropic transitions are of the same magnitude as those presented in literature for other main chain thermotropic liquid crystalline polyesters. The values associated with the isotropisation are somewhat on the higher side. Similar high values have been noted in methyl substituted series of polyesters as well. ${ }^{29}$ The origin of such high values indicates that some conformational ordering of spacers in the liquid crystalline state.

The higher values of entropy change (Tables 2 and 3) for liquid crystal to isotropization temperature for the polyesters having chloro substituent on hydroquinone as central moiety in triad based mesogenic diol suggesting poor thermal stability of mesophase and also indicating the existence of a higher degree of order in their liquid crystalline state. ${ }^{30}$

\section{Conclusions}

In this study, thermotropic liquid crystalline polyesters with very large mesophasic stability $\left(>123^{\circ} \mathrm{C}\right)$ were generated by incorporating a chloro substitution on triad mesogen. These polyesters display somewhat complex nematic ordering in the liquid crystalline state. Smectic liquid crystalline phase manifests with decamethylene spacer $(\mathrm{n}=10)$. The weak odd-even effect is noted in transition temperatures as well as in enthalpy and entropy values. The high enthalpy and entropy changes on isotropisation indicate conformational ordering in the liquid crystalline state. Unlike in random copolyesters, incorporation of chloro substituent into an ordered polyester structure depresses the isotropisation temperature.

\section{Supplementary Information (SI)}

The characterization details including IR, ${ }^{1} \mathrm{H}$ and ${ }^{13} \mathrm{C}$ NMR, thermogravimetric analysis (TGA) and wide angle $\mathrm{X}$-ray diffractogram of triad based mesogenic diol BHBOCB are presented in Supplementary Information which is available at www.ias.ac.in/chemsci.

\section{References}

1. Han H and Bhowmik P K 1997 Wholly aromatic liquidcrystalline polyesters Prog. Polym. Sci. 221431

2. (a) Chavan N N and Mulani K B 2012 In Thermotropic liquid crystalline polyesters (Germany: Lambert Academic Publishing Co.); (b) Jin J I and Kang C S 1997 Thermotropic main-chain polyesters Prog. Polym. Sci. 22937

3. Negi Y S and Goyal R K 2003 Development of thermotropic liquid crystalline polymers and their applications Int. J. Plastics Technol. 799

4. Broer D J, Lub J and Mol G N 1995 Wide-band reflective polarizers from cholesteric polymer networks with a pitch gradient Nature $\mathbf{3 7 8} 467$

5. Bunning T J and Kreuzer F H 1995 Cyclosiloxane-based liquid crystalline materials Trends Polym. Sci. 3318

6. Xing C, Jacky W Y, Zhao K Q and Tang B Z2008 Synthesis and liquid crystalline properties of poly(1-alkyne)s 
carrying triphenylene discogens J. Polym. Sci. Part A: Polym. Chem. 462960

7. Zhu Z, Zhi J, Liu A, Cui J, Tang H, Qiao W, Wan $\mathrm{X}$ and Zhou Q 2007 Synthesis and characterization of a thermotropic liquid-crystalline poly[2,5-bis(4-alkoxy carbonyl phenyl)styrene] J. Polym. Sci. Part A: Polym. Chem. $\mathbf{4 5} 830$

8. Luzny W, Pomarzanska S E and Pron A 1999 Structural properties of selected poly(azomethines) Polymer 406611

9. Shiota A and Ober C K 1997 Rigid rod liquid crystalline thermosets Prog. Polym. Sci. 22975

10. Ortiz C, Wagner M, Bhargava N, Ober C K and Kramer E J 1998 Deformation of a polydomain, smectic liquid crystalline elastomer Macromolecules 318531

11. Ortiz C, Ober C K and Kramer E J 1998 Stress relaxation of a main-chain, smectic, polydomain liquid crystalline elastomer Polymer 393713

12. Heino M T, Hietaoja P T, Vainio T P and Seppala J V 1994 Effect of viscosity ratio and processing conditions on the morphology of blends of liquid crystalline polymer and polypropylene J. Appl. Polym. Sci. 51259

13. Bualek-Limcharoen S, Saengsuwan S, Amornsakchai T and Wanno B 2001 Rheology, Morphology and tensile properties of thermotropic liquid crystalline polymer/polypropylene in-situ composites Macromol. Symp. 170189

14. Zhou Q and Lenz R W 1983 Liquid crystalline prolymers. 15. Synthesis and liquid crystalline properties of alkyl-substituted polyesters J. Polym. Sci. Part A: Polym. Chem. 213313

15. Kleinschuster J J, Pletcher T C, Schaefgen J R and Luise R R 1994 Ger. Offen. 2520

16. Roviello A and Sirigu A 1975 Mesophasic structures in polymers. A preliminary account on the mesophases of some polyalkanoates of $\mathrm{p}, \mathrm{p}^{\prime}$-dihydroxy- $\alpha, \boldsymbol{\alpha}^{\prime}$ dimethyl dibenalazine J. Polym. Sci. Polym. Lett. Ed. 13455

17. Mulani K, Momin M, Ganjave N and Chavan N 2014 Thermotropic liquid crystalline polyesters based on trimesogenic diol and flexible spacers Malaysian Polym. J. 954

18. Mulani K, Momin M, Ganjave N and Chavan N 2015 Thermotropic liquid crystalline polyesters derived from bis-(4-hydroxybenzoyloxy)-2-methyl-1,4-benzene and aliphatic dicarboxylic acid chlorides Bull. Mater. Sci. 381301
19. Mulani K, Ganjave N and Chavan N 2014 Synthesis and characterization of triad based rigid mesogenic diols derived from hydroquinone and 4-hydroxybenzoic acid Ind. J. Chem. B 53B 591

20. Pereira F V, Borsali R, Ritter O M S, Gonçalves P F, Merloa A A and da Silveira N P 2006 Structure-property relationships of smectic liquid crystalline polyacrylates as revealed by SAXS J. Braz. Chem. Soc. 17333

21. Gray G W and Warrall B M 1959 The mesomorphic transition temperature of 3-substituted 4-noctyloxydiphenyl-4-carboxilic acids J. Chem. Soc. 1545

22. Gray G W, Johns B and Marson F 1957 Mesomorphism and chemical constitution. Part VIII. The effect of 3'-substituents on the mesomorphism of the 4'-nalkyloxyphenyl-4-carboxylic acids and their alkyl esters J. Chem. Soc. 393

23. Branch S J, Byron D J, Gray G W, Ibbotson A and Warrall B M 1964 Mesomorphism and chemical constitution. Part XII. The preparation and mesomorphic properties of substituted 4, 4'-di-(p-n-alkoxybenzylideneamino) biphenyls J. Chem. Soc. 3279

24. Arora S L, Ferguson J L and Taylor T R 1970 Molecular structure and liquid crystallinity phenylene Bis(alkoxybenzoates) J. Org. Chem. 354055

25. Young W R, Haller I and Green D C 1972 Mesomorphic structure of some ring-methylated phenyl benzoyloxybenzoates J. Chem. Soc. 373707

26. Sonpatki M M, Ravindranath K and Ponrathnam S 1994 Segmented copoly(ether-ester) elastomers. Influence of hard segment length and substitution on mesophase formation Polym. J. 26804

27. Jin J, Chung B Y, Chol J and Jo B 1991 Inportance of the role of flexible spacers in liquid crystal formation by bend dimesogenic and star-shaped trimesogenic compounds Bull. Korean Chem. Soc. 12189

28. Meurisse P, Noel C, Monnerie L and Fayolle B 1981 Polymers with mesogenic elements and flexible spacers in the main chain: Aromatic-aliphatic polyesters British Polymer J. 1355

29. Lenz R W 1985 Synthesis and properties of thermotropic liquid crystal polymers with main chain mesogenic units Polymer J. 17105

30. Jo B W, Lenz R W and Jin J 1982 Liquid crystalline polymers, 7 . Thermotropic polyesters with main chain phenyl-1,4-phenylene,4,4'-biphenlene, and 1,1'binaphthyl-4,4'-ylene units Makromol. Chem. Rapid Comm. 323 